\title{
Growth Estimates for Analytic Vector-Valued Functions in the Unit Ball Having Bounded L-index in Joint Variables
}

\author{
VITA BAKSA, ANDRIY BANDURA*, AND OLEH SKASKIV
}

\begin{abstract}
Our results concern growth estimates for vector-valued functions of $\mathbb{L}$-index in joint variables which are analytic in the unit ball. There are deduced analogs of known growth estimates obtained early for functions analytic in the unit ball. Our estimates contain logarithm of sup-norm instead of logarithm modulus of the function. They describe the behavior of logarithm of norm of analytic vector-valued function on a skeleton in a bidisc by behavior of the function $\mathbf{L}$. These estimates are sharp in a general case. The presented results are based on bidisc exhaustion of a unit ball.
\end{abstract}

Keywords: Bounded index, bounded L-index in joint variables, analytic function, unit ball, growth estimates, maximum norm.

2010 Mathematics Subject Classification: 32A10, 32A17, 32A37.

\section{INTRODUCTION}

In this paper, we consider vector-valued functions of bounded $\mathbf{L}$-index in joint variables which are analytic in the unit ball. This paper is a continuation of investigations initiated in $[1,2,3]$. There was proposed the definition of $\mathbf{L}$-index boundedness in joint variables and obtained some criteria of $\mathbf{L}$-index boundedness in joint variables for vector-valued analytic functions in the unit ball.

Here, we pose the following goal: to obtain growth estimates of analytic functions having bounded L-index in joint variables. It is important because functions of bounded index has many applications in analytic theory of linear differential equations. Moreover, vector-valued entire functions of bounded index in joint variables have applications to some system of partial differential equations [19]. Therefore, combination of sufficient conditions of $L$-index boundedness for analytic solutions of the system with growth estimates of functions from this class will give a priori estimates of growth for all analytical solutions of the system.

Other applications of concept of bounded index in analytic theory of differential equations were considered for various function classes: entire functions of bounded $L$-index in direction [12], entire functions of bounded L-index in joint variables [15], analytic functions in the unit ball having bounded L-index in joint variables [4], entire bivariate vector-valued function of bounded index [19]. 


\section{NOTATIONS, DEFINITIONS AND AUXILIARY PROPOSITIONS}

We need some standard notations (for example see $[5,4,6])$. Let $\mathbb{R}_{+}=[0 ;+\infty), \mathbf{0}=(0,0) \in$ $\mathbb{R}_{+}^{2}, \mathbf{1}=(1,1) \in \mathbb{R}_{+}^{2}, R=\left(r_{1}, r_{2}\right) \in \mathbb{R}_{+}^{2},|(z, \omega)|=\sqrt{|z|^{2}+|\omega|^{2}}$. For $A=\left(a_{1}, a_{2}\right) \in \mathbb{R}^{2}$, $B=\left(b_{1}, b_{2}\right) \in \mathbb{R}^{2}$, we will use formal notations without violation of the existence of these expressions: $A B=\left(a_{1} b_{1}, a_{2} b_{2}\right), A / B=\left(a_{1} / b_{1}, a_{2} / b_{2}\right), A^{B}=\left(a_{1}^{b_{1}}, a_{2}^{b_{2}}\right)$, and the notation $A<B$ means that $a_{j}<b_{j}, j \in\{1,2\}$; the relation $A \leq B$ is defined in the similar way. For $K=$ $\left(k_{1}, k_{2}\right) \in \mathbb{Z}_{+}^{2}$, let us denote $K !=k_{1} ! \cdot k_{2}$ !. Addition, multiplication by scalar and conjugation in $\mathbb{C}^{2}$ is defined componentwise. For $z \in \mathbb{C}^{2}, w \in \mathbb{C}^{2}$ we define $\langle z, w\rangle=z_{1} \bar{w}_{1}+z_{2} \bar{w}_{2}$, where $\bar{w}_{1}, \bar{w}_{2}$ is the complex conjugate of $w_{1}, w_{2}$.

The bidisc $\left\{(z, \omega) \in \mathbb{C}^{2}:\left|z-z_{0}\right|<r_{1},\left|\omega-\omega_{0}\right|<r_{2}\right\}$ is denoted by $\mathbb{D}^{2}\left(\left(z_{0}, \omega_{0}\right), R\right)$, its skeleton $\left\{(z, \omega) \in \mathbb{C}^{2}:\left|z-z_{0}\right|=r_{1},\left|\omega-\omega_{0}\right|=r_{2}\right\}$ is denoted by $\mathbb{T}^{2}\left(\left(z_{0}, \omega_{0}\right), R\right)$, the closed polydisc $\left\{(z, \omega) \in \mathbb{C}^{2}:\left|z-z_{0}\right| \leq r_{1},\left|\omega-\omega_{0}\right| \leq r_{2}\right\}$ is denoted by $\mathbb{D}^{2}\left[\left(z_{0}, \omega_{0}\right), R\right], \mathbb{D}^{2}=\mathbb{D}^{2}(\mathbf{0} ; \mathbf{1})$, $\mathbb{D}=\{z \in \mathbb{C}:|z|<1\}$. The open ball $\left\{(z, \omega) \in \mathbb{C}^{2}: \sqrt{\left|z-z_{0}\right|^{2}+\left|\omega-\omega_{0}\right|^{2}}<r\right\}$ is denoted by $\mathbb{B}^{2}\left(\left(z_{0}, \omega_{0}\right), r\right)$, the sphere $\left\{(z, \omega) \in \mathbb{C}^{2}: \sqrt{\left|z-z_{0}\right|^{2}+\left|\omega-\omega_{0}\right|^{2}}=r\right\}$ is denoted by $\mathbb{S}^{2}\left(\left(z_{0}, \omega_{0}\right), r\right)$, and the closed ball $\left\{z \in \mathbb{C}^{2}: \sqrt{\left|z-z_{0}\right|^{2}+\left|\omega_{0}-\omega_{0}\right|^{2}} \leq r\right\}$ is denoted by $\mathbb{B}^{2}\left[\left(z_{0}, \omega_{0}\right), r\right], \mathbb{B}^{2}=\mathbb{B}^{2}(\mathbf{0}, \mathbf{1}), \mathbb{D}=\mathbb{B}^{1}=\{z \in \mathbb{C}:|z|<1\}$.

Let $F(z, \omega)=\left(f_{1}(z, \omega), f_{2}(z, \omega)\right)$ be an analytic vector-function in $\mathbb{B}^{2}$. Then at a point $(a, b) \in$ $\mathbb{B}^{2}$, the function $F(z, \omega)$ has a bivariate Taylor expansion:

$$
F(z, \omega)=\sum_{k=0}^{\infty} \sum_{m=0}^{\infty} C_{k l}(z-a)^{k}(\omega-b)^{m},
$$

where $C_{k m}=\left.\frac{1}{k ! m !}\left(\frac{\partial^{k+m} f_{1}(z, \omega)}{\partial z^{k} \partial \omega^{m}}, \frac{\partial^{k+m} f_{2}(z, \omega)}{\partial z^{k} \partial \omega^{m}}\right)\right|_{z=a, \omega=b}=\frac{1}{k ! m !} F^{(k, m)}(a, b)$.

Let $\mathbf{L}(z, \omega)=\left(l_{1}(z, \omega), l_{2}(z, \omega)\right)$, where $l_{j}(z, \omega): \mathbb{B}^{2} \rightarrow \mathbb{R}_{+}^{2}$ is a positive continuous function such that

$$
\forall(z, \omega) \in \mathbb{B}^{2}: \quad l_{j}(z, \omega)>\frac{\beta}{1-\sqrt{|z|^{2}+|\omega|^{2}}},
$$

$j \in\{1,2\}$, where $\beta>\sqrt{2}$ is a some constant.

The norm for the vector-function $F: \mathbb{B}^{2} \rightarrow \mathbb{C}^{2}$ is defined as the sup-norm:

$$
\|F(z, \omega)\|=\max _{1 \leq j \leq 2}\left\{\left|f_{j}(z, \omega)\right|\right\} .
$$

We write

$$
F^{(i, j)}(z, \omega)=\frac{\partial^{i+j} F(z, \omega)}{\partial z^{i} \partial \omega^{j}}=\left(\frac{\partial^{i+j} f_{1}(z, \omega)}{\partial z^{i} \partial \omega^{j}}, \frac{\partial^{i+j} f_{2}(z, \omega)}{\partial z^{i} \partial \omega^{j}}\right) .
$$

An analytic vector-function $F: \mathbb{B}^{2} \rightarrow \mathbb{C}^{2}$ is said to be of bounded L-index (in joint variables), if there exists $n_{0} \in \mathbb{Z}_{+}$such that

$$
\frac{\left\|F^{(i, j)}(z, \omega)\right\|}{i ! j ! l_{1}^{i}(z, \omega) l_{2}^{j}(z, \omega)} \leq \max \left\{\frac{\left\|F^{(k, m)}(z, \omega)\right\|}{k ! m ! l_{1}^{k}(z, \omega) l_{2}^{m}(z, \omega)}: k, m \in \mathbb{Z}_{+}, k+m \leq n_{0}\right\} .
$$

The least such integer $n_{0}$ is called the $\mathbf{L}$-index in joint variables of the vector-function $F$ and is denoted by $N\left(F, \mathbf{L}, \mathbb{B}^{2}\right)$. The concept of boundedness of $\mathbf{L}$-index in joint variables were considered for other classes of analytic functions. They are differed domains of analyticity: the unit ball $[5,4,11,13]$, the polydisc $[8,10]$, the Cartesian product of the unit disc and complex plane [9], $n$-dimensional complex space [7, 11, 14]. Vector-valued functions of one and several complex variables having bounded index were considered in [18, 20, 17, 23, 21, 19]. 
The function class $Q\left(\mathbb{B}^{2}\right)$ is defined as following: $\forall R \in \mathbb{R}_{+}^{2},|R| \leq \beta, j \in\{1,2\}$ :

$$
0<\lambda_{1, j}(R) \leq \lambda_{2, j}(R)<\infty,
$$

where

$$
\begin{aligned}
& \lambda_{1, j}(R)=\inf _{\left(z_{0}, \omega_{0}\right) \in \mathbb{B}^{2}} \inf \left\{\frac{l_{j}(z, \omega)}{l_{j}\left(z_{0}, \omega_{0}\right)}:(z, \omega) \in \mathbb{D}^{2}\left[\left(z_{0}, \omega_{0}\right), R / \mathbf{L}\left(z_{0}, \omega_{0}\right)\right]\right\}, \\
& \lambda_{2, j}(R)=\sup _{\left(z_{0}, \omega_{0}\right) \in \mathbb{B}^{2}} \sup \left\{\frac{l_{j}(z, \omega)}{l_{j}\left(z_{0}, \omega_{0}\right)}:(z, \omega) \in \mathbb{D}^{2}\left[\left(z_{0}, \omega_{0}\right), R / \mathbf{L}\left(z_{0}, \omega_{0}\right)\right]\right\} .
\end{aligned}
$$

We need some propositions from $[1,2]$.

For an analytic vector-function $F: \mathbb{B}^{2} \rightarrow \mathbb{C}^{2}$, we put

$$
M\left(R,\left(z_{0}, \omega_{0}\right), F\right)=\max \left\{\|F(z, \omega)\|:(z, \omega) \in \mathbb{T}^{2}\left(\left(z_{0}, \omega_{0}\right), R\right)\right\},
$$

where $\left(z_{0}, \omega_{0}\right) \in \mathbb{B}^{2}, R \in \mathbb{R}_{+}^{2}$. Then

$$
M\left(R,\left(z_{0}, \omega_{0}\right), F\right)=\max \left\{\|F(z, \omega)\|:(z, \omega) \in \mathbb{D}^{2}\left(\left(z_{0}, \omega_{0}\right), R\right)\right\},
$$

because the maximum modulus of the analytic vector-function in a closed bidisc is attained on its skeleton.

To prove an growth estimates, we need the following theorem. The theorem gives sufficient conditions by the estimate of maximum modulus on the skeleton of bidisc.

Theorem 2.1 ([2]). Let $\mathbf{L} \in Q\left(\mathbb{B}^{2}\right)$. If analytic vector-function $F: \mathbb{B}^{2} \rightarrow \mathbb{C}^{2}$ has bounded $\mathbf{L}$-index in joint variables, then for all $R^{\prime}, R^{\prime \prime} \in \mathbb{R}_{+}^{2}, R^{\prime}<R^{\prime \prime},\left|R^{\prime \prime}\right| \leq \beta$ there exists $p_{1}=p_{1}\left(R^{\prime}, R^{\prime \prime}\right) \geq 1$ such that for every $\left(z_{0}, \omega_{0}\right) \in \mathbb{B}^{2}$ inequality

$$
M\left(\frac{R^{\prime \prime}}{\mathbf{L}\left(z_{0}, \omega_{0}\right)},\left(z_{0}, \omega_{0}\right), F\right) \leq p_{1} M\left(\frac{R^{\prime}}{\mathbf{L}\left(z_{0}, \omega_{0}\right)},\left(z_{0}, \omega_{0}\right), F\right)
$$

holds.

\section{GROWTH ESTIMATES OF ANALYTIC VECTOR-VALUED FUNCTIONS IN THE UNIT BALL}

We put $[0,2 \pi]^{2}=[0,2 \pi] \times[0,2 \pi]$. For $R=\left(r_{1}, r_{2}\right) \in \mathbb{R}_{+}^{2}, \Theta=\left(\theta_{1}, \theta_{2}\right) \in[0,2 \pi]^{2}, A=\left(a_{1}, a_{2}\right) \in$ $\mathbb{C}^{2}$, we will write

$$
R e^{i \Theta}=\left(r_{1} e^{i \theta_{1}}, r_{2} e^{i \theta_{2}}\right), \quad \arg A=\left(\arg a_{1}, \arg a_{2}\right) .
$$

Denote by $K\left(\mathbb{B}^{2}\right)$ the class of positive continuous vector-valued functions $\mathbf{L}=\left(l_{1}, l_{2}\right)$, where every $l_{j}: \mathbb{B}^{2} \rightarrow \mathbb{R}_{+}$obeys inequality (2.1) and there exists $c \geq 1$ such that for all $R \in \mathbb{R}_{+}^{2}$ with $|R|<1$ and $j \in\{1,2\}$,

$$
\max _{\Theta_{1}, \Theta_{2} \in[0,2 \pi]^{2}} \frac{l_{j}\left(R e^{i \Theta_{2}}\right)}{l_{j}\left(R e^{i \Theta_{1}}\right)} \leq c .
$$

In the case $\mathbf{L}(z, w)=\left(l_{1}(|z|,|w|), l_{2}(|z|,|w|)\right)$, we have that $\mathbf{L} \in K\left(\mathbb{B}^{2}\right)$. Put $\boldsymbol{\beta}=\left(\frac{\beta}{\sqrt{2}}, \frac{\beta}{\sqrt{2}}\right)$. 
Theorem 3.2. Let $\mathbf{L} \in Q\left(\mathbb{B}^{2}\right) \bigcap K\left(\mathbb{B}^{2}\right), \beta>c \sqrt{2}$. If an analytic vector-function $F: \mathbb{B}^{2} \rightarrow \mathbb{C}^{2}$ has bounded $\mathbf{L}$-index in joint variables, then

$$
\begin{aligned}
& \ln \max \left\{|F(z, w)|:(z, w) \in \mathbb{T}^{2}(\mathbf{0}, R)\right\}= \\
& =O\left(\operatorname { m i n } \left\{\min _{\Theta \in[0,2 \pi]^{2}}\left(\int_{0}^{r_{1}} l_{1}\left(t e^{i \theta_{1}}, r_{2} e^{i \theta_{2}}\right) d t+\int_{0}^{r_{2}} l_{2}\left(r_{1}^{0}, t\right) d t\right)\right.\right. \text {; } \\
& \left.\left.\min _{\Theta \in[0,2 \pi]^{2}}\left(\int_{0}^{r_{1}} l_{1}\left(t e^{i \theta_{1}}, r_{2} e^{i \theta_{2}}\right) d t+\int_{0}^{r_{2}} l_{2}\left(r_{1}^{0}, t\right) d t\right)\right\}\right),
\end{aligned}
$$

with $|R| \rightarrow 1-0, R^{0}=\left(r_{1}^{0}, r_{2}^{0}\right)$ is a fixed radius.

Proof. Let $R>0,|R|>1, \Theta \in[0,2 \pi]^{2}$, and a point $\left(z^{*}, w^{*}\right) \in \mathbb{T}^{2}\left(\mathbf{0}, R+\frac{\boldsymbol{\beta}}{\mathbf{L}\left(R e^{i \ominus}\right)}\right)$ be such that

$$
\left\|F\left(z^{*}, w^{*}\right)\right\|=\max \left\{\|F(z, w)\|:(z, w) \in \mathbb{T}^{2}\left(\mathbf{0}, R+\frac{\boldsymbol{\beta}}{\mathbf{L}\left(R e^{i \Theta}\right)}\right)\right\} .
$$

We put $z_{0}=\frac{z^{*} r_{1}}{R+\boldsymbol{\beta} / \mathbf{L}\left(R e^{i \odot}\right)}, w_{0}=\frac{w^{*} r_{2}}{R+\boldsymbol{\beta} / \mathbf{L}\left(R e^{i \odot}\right)}$. Thus,

$$
\begin{gathered}
\left|z_{0}-z^{*}\right|=\left|\frac{z^{*} r_{1}}{r_{1}+\frac{\beta}{c \sqrt{2} l_{1}\left(R e^{i \Theta}\right)}}-z^{*}\right|=\left|\frac{z^{*} \beta /\left(c \sqrt{2} l_{1}\left(R e^{i \Theta}\right)\right)}{r_{1}+\frac{\beta}{c \sqrt{2} l_{1}\left(R e^{i \Theta}\right)}}\right|=\frac{\beta}{c \sqrt{2} l_{1}\left(R e^{i \Theta}\right)}, \\
\left|w_{0}-w^{*}\right|=\left|\frac{w^{*} r_{2}}{r_{2}+\frac{\beta}{c \sqrt{2} l_{2}\left(R e^{i \Theta}\right)}}-w^{*}\right|=\left|\frac{w^{*} \beta /\left(c \sqrt{2} l_{2}\left(R e^{i \Theta}\right)\right)}{r_{2}+\frac{\beta}{c \sqrt{2} l_{2}\left(R e^{i \Theta}\right)}}\right|=\frac{\beta}{c \sqrt{2} l_{2}\left(R e^{i \Theta}\right)}, \\
\quad \mathbf{L}\left(z_{0}, w_{0}\right)=\mathbf{L}\left(\frac{z^{*} r_{1}}{R+\boldsymbol{\beta} / \mathbf{L}\left(R e^{i \Theta}\right)}, \frac{w^{*} r_{2}}{R+\boldsymbol{\beta} / \mathbf{L}\left(R e^{i \Theta}\right)}\right)= \\
=\mathbf{L}\left(\frac{\left(R+\boldsymbol{\beta} / \mathbf{L}\left(R e^{i \Theta}\right)\right) r_{1} e^{i \arg z^{*}}}{R+\boldsymbol{\beta} / \mathbf{L}\left(R e^{i \Theta}\right)}, \frac{\left(R+\boldsymbol{\beta} / \mathbf{L}\left(R e^{i \Theta}\right)\right) r_{2} e^{i \arg w^{*}}}{R+\boldsymbol{\beta} / \mathbf{L}\left(R e^{i \Theta}\right)}\right)= \\
=\mathbf{L}\left(r_{1} e^{i \arg z^{*}}, r_{2} e^{i \arg w^{*}}\right) .
\end{gathered}
$$

Since $\mathbf{L} \in K\left(\mathbb{B}^{2}\right)$, we have

$$
c \mathbf{L}\left(z_{0}, w_{0}\right)=c \mathbf{L}\left(r_{1} e^{i \arg z^{*}}, r_{2} e^{i \arg w^{*}}\right) \geq \mathbf{L}\left(r_{1} e^{i \theta_{1}}, r_{2} e^{i \theta_{2}}\right) \geq \frac{1}{c} \mathbf{L}\left(z_{0}, w_{0}\right) .
$$

We will consider two skeletons $\mathbb{T}^{2}\left(\left(z_{0}, w_{0}\right), \frac{\mathbf{e}}{\mathbf{L}\left(z_{0}, w_{0}\right)}\right)$ and $\mathbb{T}^{2}\left(\left(z_{0}, w_{0}\right), \frac{\boldsymbol{\beta}}{\mathbf{L}\left(z_{0}, w_{0}\right)}\right)$. By Theorem 2.1 , there exist $p_{1}=p_{1}\left(\frac{\mathrm{e}}{c}, c \boldsymbol{\beta}\right) \geq 1$ such that (2.5) is true for $R^{\prime}=\frac{\mathrm{e}}{c}, R^{\prime \prime}=c \boldsymbol{\beta}$ :

$$
\begin{aligned}
& \max \left\{\|F(z, w)\|:(z, w) \in \mathbb{T}^{2}\left(\mathbf{0}, R+\frac{\boldsymbol{\beta}}{\mathbf{L}\left(r_{1} e^{i \theta_{1}}, r_{2} e^{i \theta_{2}}\right)}\right)\right\} \leq \\
& \leq\left\{\|F(z, w)\|:(z, w) \in \mathbb{T}^{2}\left(\left(z_{0}, w_{0}\right), \frac{\boldsymbol{\beta}}{\mathbf{L}\left(r_{1} e^{i \theta_{1}}, r_{2} e^{i \theta_{2}}\right)}\right)\right\} \leq \\
& \quad \leq\left\{\|F(z, w)\|:(z, w) \in \mathbb{T}^{2}\left(\left(z_{0}, w_{0}\right), \frac{c \boldsymbol{\beta}}{\mathbf{L}\left(z_{0}, w_{0}\right)}\right)\right\} \leq \\
& \leq p_{1}\left\{\|F(z, w)\|:(z, w) \in \mathbb{T}^{2}\left(\left(z_{0}, w_{0}\right), \frac{\mathbf{e}}{c \mathbf{L}\left(z_{0}, w_{0}\right)}\right)\right\} \leq \\
& \leq p_{1}\left\{\|F(z, w)\|:(z, w) \in \mathbb{T}^{2}\left(\mathbf{0}, R+\frac{\mathbf{e}}{\mathbf{L}\left(r_{1} e^{i \theta_{1}}, r_{2} e^{i \theta_{2}}\right)}\right)\right\} .
\end{aligned}
$$


The function $\ln ^{+} \max \left\{\|F(z, w)\|:(z, w) \in \mathbb{T}^{2}(\mathbf{0}, R)\right\}$ is convex relative $\ln r_{1}, \ln r_{2}$. Therefore,

$$
\begin{gathered}
\ln ^{+} \max \left\{\|F(z, w)\|:(z, w) \in \mathbb{T}^{2}(\mathbf{0}, R)\right\}- \\
-\ln ^{+} \max \left\{\|F(z, w)\|:(z, w) \in \mathbb{T}^{2}\left(\mathbf{0}, R+\left(r_{1}^{0}-r_{1}\right) e_{1}\right)\right\}=\int_{r_{1}^{0}}^{r_{1}} \frac{A_{1}\left(t, r_{2}\right)}{t} d t, \\
\ln ^{+} \max \left\{\|F(z, w)\|:(z, w) \in \mathbb{T}^{2}(\mathbf{0}, R)\right\}- \\
-\ln ^{+} \max \left\{\|F(z, w)\|:(z, w) \in \mathbb{T}^{2}\left(\mathbf{0}, R+\left(r_{2}^{0}-r_{2}\right) e_{2}\right)\right\}=\int_{r_{2}^{0}}^{r_{2}} \frac{A_{2}\left(r_{1}, t\right)}{t} d t
\end{gathered}
$$

for each $0<r_{j}^{0}<r_{j}, j\{1,2\}$, where functions $A_{1}\left(t, r_{2}\right), A_{2}\left(r_{1}, t\right)$ are positive non-decreasing $t$. Then from (3.7), we obtain

$$
\begin{aligned}
& \ln p_{1} \geq \ln \max \left\{\|F(z, w)\|:(z, w) \in \mathbb{T}^{2}\left(\mathbf{0}, R+\frac{\boldsymbol{\beta}}{\mathbf{L}\left(R e^{i \Theta}\right)}\right)\right\}- \\
& -\ln \max \left\{\|F(z, w)\|:(z, w) \in \mathbb{T}^{2}\left(\mathbf{0}, R+\frac{\mathbf{e}}{\mathbf{L}\left(R e^{i \Theta}\right)}\right)\right\}= \\
& =\ln \max \left\{\|F(z, w)\|:(z, w) \in \mathbb{T}^{2}\left(\mathbf{0}, R+\frac{\mathbf{e}+\left(\frac{\beta}{\sqrt{2} c}-1\right) \mathbf{e}_{1}}{\mathbf{L}\left(R e^{i \Theta}\right)}\right)\right\}- \\
& -\ln \max \left\{\|F(z, w)\|:(z, w) \in \mathbb{T}^{2}\left(\mathbf{0}, R+\frac{\mathbf{e}+\left(\frac{\beta}{\sqrt{2} c}-1\right) \mathbf{e}_{2}}{\mathbf{L}\left(R e^{i \Theta}\right)}\right)\right\}= \\
& =\int_{r_{1}+1 / l_{1}\left(R e^{i \Theta}\right)}^{r_{1}+\beta /\left(c \sqrt{2} l_{1}\left(R e^{i \Theta}\right)\right)} \frac{1}{t} A_{1}\left(t, r_{2}+\frac{\beta}{c \sqrt{2} l_{2}\left(R e^{i \Theta}\right)}\right) d t+ \\
& +\int_{r_{2}+1 / l_{2}\left(R e^{i \Theta}\right)}^{r_{2}+\beta /\left(c \sqrt{2} l_{2}\left(R e^{i \Theta}\right)\right)} \frac{1}{t} A_{2}\left(r_{1}+\frac{\beta}{c \sqrt{2} l_{1}\left(R e^{i \Theta}, t\right)}\right) d t \geq \\
& \geq \ln \left(1+\frac{\frac{\beta}{\sqrt{2} c}-1}{r_{1} l_{1}\left(R e^{i \Theta}\right)+1}\right) A_{1}\left(r_{1}, r_{2}+\frac{1}{l_{2}\left(R e^{i \Theta}\right)}\right)+\ln \left(1+\frac{\frac{\beta}{\sqrt{2} c}-1}{r_{2} l_{2}\left(R e^{i \Theta}\right)+1}\right) \times \\
& \times A_{2}\left(r_{1}+\frac{1}{l_{1}\left(R e^{i \Theta}\right)}, r_{2}\right) .
\end{aligned}
$$

Then, we have $r_{j} l_{j}\left(R e^{i \Theta}\right) \longrightarrow+\infty$ with $|R| \longrightarrow 1-0$. We obtain, for $j \in\{1,2\}$ and $r_{j} \geq r_{j}^{0}$ :

$$
\ln \left(1+\frac{\frac{\beta}{\sqrt{2} c}-1}{r_{j} l_{j}\left(R e^{i \Theta}\right)+1}\right) \sim \frac{\frac{\beta}{\sqrt{2} c}-1}{r_{j} l_{j}\left(R e^{i \Theta}\right)+1} \geq \frac{\frac{\beta}{\sqrt{2} c}-1}{2 r_{j} l_{j}\left(R e^{i \Theta}\right)} .
$$

Thus, (3.10) implies that

$$
\begin{gathered}
A_{1}\left(r_{1}, r_{2}+\frac{\beta}{c \sqrt{2}} l_{2}\left(R e^{i \Theta}\right)\right) \leq \frac{2 \ln p_{1}}{\frac{\beta}{c \sqrt{2}}-1} r_{1} l_{1}\left(R e^{i \Theta}\right), \\
A_{2}\left(r_{1} \frac{\beta}{c \sqrt{2} l_{1}\left(R e^{i \Theta}\right)}, r_{2}\right) \leq \frac{2 \ln p_{1}}{\frac{\beta}{c \sqrt{2}}-1} r_{2} l_{2}\left(R e^{i \Theta}\right) .
\end{gathered}
$$


Let $R^{0}=\left(r_{1}^{0}, r_{2}^{0}\right)$, where $r_{j}^{0}$ it is chosen higher. From inequalities (3.8) and (3.9), it follows that $\ln \max \left\{\|F(z, w)\|:(z, w) \in \mathbb{T}^{2}(\mathbf{0}, R)\right\}=$

$$
\begin{aligned}
&=\ln \max \{\left.\|F(z, w)\|:(z, w) \in \mathbb{T}^{2}\left(\mathbf{0}, R+\left(r_{1}^{0}-r_{1}\right) \mathbf{e}_{1}\right)\right\}+\int_{r_{1}^{0}}^{r_{1}} \frac{A_{1}\left(t, r_{2}\right)}{t} d t= \\
&=\ln \max \left\{\|F(z, w)\|:(z, w) \in \mathbb{T}^{2}\left(\mathbf{0}, R+\left(r_{1}^{0}-r_{1}\right) \mathbf{e}_{1}+\left(r_{2}^{0}-r_{2}\right) \mathbf{e}_{2}\right)\right\}+ \\
& \quad+\int_{r_{1}^{0}}^{r_{1}} \frac{A_{1}\left(t, r_{2}\right)}{t} d t+\int_{r_{2}^{0}}^{r_{2}} \frac{A_{2}\left(r_{1}^{0}, t\right)}{t} d t= \\
&=\ln \max \left\{\|F(z, w)\|:(z, w) \in \mathbb{T}^{2}\left(\mathbf{0}, R^{0}\right)\right\}+\frac{2 \ln p_{1}}{\frac{\beta}{\sqrt{2} c}-1} \times \\
& \quad \times\left(\int_{0}^{r_{1}} l_{1}\left(t e^{i \theta_{1}}, r_{2} e^{i \theta_{2}}\right) d t+\int_{0}^{r_{2}} l_{2}\left(r_{1}^{0} e^{i \theta_{1}}, t e^{i \theta_{2}}\right) d t\right) \leq \\
& \leq(1+O(1)) \frac{2 \ln p_{1}}{\frac{\beta}{c \sqrt{2}}-1}\left(\int_{0}^{r_{1}} l_{1}\left(t e^{i \theta_{1}}, r_{2} e^{i \theta_{2}}\right) d t+\int_{0}^{r_{2}} l_{2}\left(r_{1}^{0} e^{i \theta_{1}}, t e^{i \theta_{2}}\right) d t\right) .
\end{aligned}
$$

Function $\ln \max \left\{\|F(z, w)\|:(z, w) \in \mathbb{T}^{2}(\mathbf{0}, R)\right\}$ is independent of $\Theta$. We obtain

$$
\begin{gathered}
\ln \max \left\{\|F(z, w)\|:(z, w) \in \mathbb{T}^{2}(\mathbf{0}, R)\right\}= \\
=O\left(\min _{\Theta \in[0,2 \pi]^{2}}\left(\int_{0}^{r_{1}} l_{1}\left(t e^{i \theta_{1}}, r_{2} e^{i \theta_{2}}\right) d t+\int_{0}^{r_{2}} l_{2}\left(r_{1}^{0} e^{i \theta_{1}}, t e^{i \theta_{2}}\right) d t\right),\right.
\end{gathered}
$$

with $|R| \longrightarrow 1-0$. Theorem is proved.

Corollary 3.1. If $\mathbf{L} \in Q\left(\mathbb{B}^{2}\right) \bigcap K\left(\mathbb{B}^{2}\right), \min _{\Theta \in[0,2 \pi]^{2}} l_{j}\left(R e^{i \Theta}\right)$ is non-decreasing in each variable $r_{k}$, $k \in\{1,2\}, j \in\{1,2\}, k \neq j$, an analytic vector-function $F: \mathbb{B}^{2} \rightarrow \mathbb{C}^{2}$ has bounded $L$-index in joint variables, then

$$
\ln \max \left\{\|F(z, w)\|:(z, w) \in \mathbb{T}^{2}(\mathbf{0}, R)\right\}=O\left(\min _{\Theta \in[0,2 \pi]^{2}} \sum_{j=1}^{2} \int_{0}^{r_{j}} l_{j}\left(R^{(j)} e^{i \Theta}\right) d t\right),
$$

as $|R| \longrightarrow 1-0$, with $R^{(1)}=\left(t, r_{2}\right), R^{(2)}=\left(r_{1}, t\right)$.

We denote $a^{+}=\max \{a, 0\}, u_{j}(t)=u_{j}(t, R, \Theta)=l_{j}\left(\frac{t R}{r^{*}} e^{i \Theta}\right)$, with $a \in \mathbb{R}, t \in \mathbb{R}_{+}, j \in\{1,2\}$, $r^{*}=\max _{1 \leq j \leq 2} r_{j} \neq 0$ and $\frac{t}{r^{*}}|R|<1$.

Theorem 3.3. Let $\mathbf{L}\left(R e^{i \Theta}\right)$ be a positive continuously differentiable function in each variable $r_{k}, k \in$ $\{1,2\},|R|<1, \Theta \in[0,2 \pi]^{2}$. If the function $L$ obeys inequality (2.1) and an analytic vector-function $F: \mathbb{B}^{2} \rightarrow \mathbb{C}^{2}$ has bounded $\mathbf{L}$-index in joint variables, then for each $\Theta \in[0,2 \pi]^{2}$ and for all $R \in \mathbb{R}_{+}^{2}$, $|R|<1$ and $(s, p) \in \mathbb{Z}^{2}$,

$$
\begin{gathered}
\ln \max \left\{\frac{\left\|F^{(s, p)}\left(R e^{i \Theta}\right)\right\|}{s ! p ! l_{1}^{s}\left(R e^{i \Theta}\right) l_{2}^{p}\left(R e^{i \Theta}\right)}: s+p \leq N\right\} \leq \\
\leq \ln \max \left\{\frac{\left\|F^{(s, p)}(\mathbf{0})\right\|}{s ! p ! l_{1}^{s}(\mathbf{0}) l_{2}^{p}(\mathbf{0})}: s+p \leq N\right\}+ \\
\int_{0}^{r^{*}}\left(\max _{s+p \leq N}\left\{(s+1) l_{1}\left(\frac{\tau}{r^{*}} R e^{i \Theta}\right)+(p+1) l_{2}\left(\frac{\tau}{r^{*}} R e^{i \Theta}\right)\right\}+\right. \\
\left.+\max _{s+p \leq N}\left\{\frac{s\left(-u_{1}^{\prime}(\tau)\right)^{+}}{l_{1}\left(\frac{\tau}{r^{*}} R e^{i \Theta}\right)}+\frac{p\left(-u_{2}^{\prime}(\tau)\right)^{+}}{l_{2}\left(\frac{\tau}{r^{*}} R e^{i \Theta}\right)}\right\}\right) d \tau .
\end{gathered}
$$


Proof. Let $R \in \mathbb{R}^{2} \backslash\{\mathbf{0}\}, \Theta \in[0,2 \pi]^{2}$. We put $a_{j}=\frac{r_{j}}{r^{*}}, j \in\{1,2\}$ and $A=\left(a_{1}, a_{2}\right)$. Consider the function

$$
g(t)=\max \left\{\frac{\left\|F^{(s, p)}\left(A e^{i \Theta}\right)\right\|}{s ! p ! l_{1}^{s}\left(A e^{i \Theta}\right) l_{2}^{p}\left(A e^{i \Theta}\right)}: s+p \leq N\right\}
$$

where $A t=\left(a_{1} t, a_{2} t\right), A t e^{i \Theta}=\left(a_{1} t^{i \theta_{1}}, a_{2} t^{i \theta_{2}}\right)$.

Since the function

$$
\frac{\left\|F^{(s, p)}\left(a_{1} e^{i \theta_{1}}, a_{2} e^{i \theta_{2}}\right)\right\|}{s ! p ! l_{1}^{s}\left(a_{1} e^{i \theta_{1}}, a_{2} e^{i \theta_{2}}\right) l_{2}^{p}\left(a_{1} e^{i \theta_{1}}, a_{2} e^{i \theta_{2}}\right)}
$$

is continuously differentiable function of real variable $t \in[0 ;+\infty)$, outside the zero set of function $\left\|F^{(s, p)}\left(a_{1} e^{i \theta_{1}}, a_{2} e^{i \theta_{2}}\right)\right\|$, then $g(t)$ is also a continuously differentiable function on $\left[0, \frac{r^{*}}{|R|}\right)$ except for a countable set of points.

Hence, in view of $\frac{d}{d r}|g(r)| \leq\left|g^{\prime}(r)\right|$, which holds everywhere except $r=t$, where $g(t)=0$ we obtain that:

$$
\begin{gathered}
\frac{d}{d t}\left(\frac{\left\|F^{(s, p)}\left(a_{1} e^{i \theta_{1}}, a_{2} e^{i \theta_{2}}\right)\right\|}{s ! p ! l_{1}^{s}\left(a_{1} e^{i \theta_{1}}, a_{2} e^{i \theta_{2}}\right) l_{2}^{p}\left(a_{1} e^{i \theta_{1}}, a_{2} e^{i \theta_{2}}\right)}\right)= \\
=\frac{1}{s ! p ! l_{1}^{s}\left(a_{1} e^{i \theta_{1}}, a_{2} e^{i \theta_{2}}\right) l_{2}^{p}\left(a_{1} e^{i \theta_{1}}, a_{2} e^{i \theta_{2}}\right)} \frac{d}{d t}\left\|F^{(s, p)}\left(a_{1} e^{i \theta_{1}}, a_{2} e^{i \theta_{2}}\right)\right\|+\left\|F^{(s, p)}\left(a_{1} e^{i \theta_{1}}, a_{2} e^{i \theta_{2}}\right)\right\| \times \\
\times \frac{d}{d t} \frac{1}{s ! p ! l_{1}^{s}\left(a_{1} e^{i \theta_{1}}, a_{2} e^{i \theta_{2}}\right) l_{2}^{p}\left(a_{1} e^{i \theta_{1}}, a_{2} e^{i \theta_{2}}\right)} \leq \frac{1}{s ! p ! l_{1}^{s}\left(a_{1} e^{i \theta_{1}}, a_{2} e^{i \theta_{2}}\right) l_{2}^{p}\left(a_{1} e^{i \theta_{1}}, a_{2} e^{i \theta_{2}}\right)} \times \\
\times\left(\left\|F^{(s+1, p)}\left(a_{1} e^{i \theta_{1}}, a_{2} e^{i \theta_{2}}\right) a_{1} e^{i \theta_{1}}\right\|+\left\|F^{(s, p+1)}\left(a_{1} e^{i \theta_{1}}, a_{2} e^{i \theta_{2}}\right) a_{2} e^{i \theta_{2}}\right\|\right)- \\
\leq \frac{\left\|F^{(s, p)}\left(a_{1} e^{i \theta_{1}}, a_{2} e^{i \theta_{2}}\right)\right\|}{s ! p ! l_{1}^{s}\left(a_{1} e^{i \theta_{1}}, a_{2} e^{i \theta_{2}}\right) l_{2}^{p}\left(a_{1} e^{i \theta_{1}}, a_{2} e^{i \theta_{2}}\right)}\left(\frac{s u_{1}^{\prime}(t)}{l_{1}\left(A t e^{i \Theta}\right)}+\frac{p u_{2}^{\prime}(t)}{l_{2}\left(A t e^{i \Theta}\right)}\right) \leq \\
+\frac{\left\|F^{(s+1, p)}\left(a_{1} e^{i \theta_{1}}, a_{2} e^{i \theta_{2}}\right)\right\|}{(s+1) ! p ! l_{1}^{s+1}\left(a_{1} e^{i \theta_{1}}, a_{2} e^{i \theta_{2}}\right) l_{2}^{p}\left(a_{1} e^{i \theta_{1}}, a_{2} e^{i \theta_{2}}\right)} a_{1}(s+1) l_{1}\left(a_{1} e^{i \theta_{1}}, a_{2} e^{i \theta_{2}}\right)+ \\
\quad \frac{\left\|F^{(s, p+1)}\left(a_{1} e^{i \theta_{1}}, a_{2} e^{i \theta_{2}}\right)\right\|}{s !(p+1) ! l_{1}^{s}\left(a_{1} e^{i \theta_{1}}, a_{2} e^{i \theta_{2}}\right) l_{2}^{p+1}\left(a_{1} e^{i \theta_{1}}, a_{2} e^{i \theta_{2}}\right)} a_{2}(p+1) l_{2}\left(a_{1} e^{i \theta_{1}}, a_{2} e^{i \theta_{2}}\right)+ \\
+\frac{\left\|F^{(s, p)}\left(a_{1} e^{i \theta_{1}}, a_{2} e^{i \theta_{2}}\right)\right\|}{s ! p ! l_{1}^{s}\left(a_{1} e^{i \theta_{1}}, a_{2} e^{i \theta_{2}}\right) l_{2}^{p}\left(a_{1} e^{i \theta_{1}}, a_{2} e^{i \theta_{2}}\right)}\left(\frac{s\left(-u_{1}^{\prime}(t)\right)^{+}}{l_{1}\left(A t e^{i \Theta}\right)}+\frac{p\left(-u_{2}^{\prime}(t)\right)^{+}}{l_{2}\left(A t e^{i \Theta}\right)}\right) .
\end{gathered}
$$


For absolutely continuous functions $h_{1}, h_{2}$ and $h(x):=\max \left\{h_{j}(z, w): 1 \leq j \leq 2\right\}$, one has $h^{\prime}(x) \leq \max \left\{h_{j}^{\prime}(z, w): 1 \leq j \leq 2\right\}, x \in[a, b]$. The function $g$ is absolutely continuous. Therefore, (3.13) implies that

$$
\begin{gathered}
g^{\prime}(t) \leq \max \left\{\frac{d}{d t}\left(\frac{\left\|F^{(s, p)}\left(a_{1} e^{i \theta_{1}}, a_{2} e^{i \theta_{2}}\right)\right\|}{s ! p ! l_{1}^{s}\left(a_{1} e^{i \theta_{1}}, a_{2} e^{i \theta_{2}}\right) l_{2}^{p}\left(a_{1} e^{i \theta_{1}}, a_{2} e^{i \theta_{2}}\right)}\right): s+p \leq N\right\} \leq \\
\leq \max _{s+p \leq N}\left\{\frac{a_{1}(s+1) l_{1}\left(A e^{i \Theta}\right)\left\|F^{(s+1, p)}\left(A e^{i \Theta}\right)\right\|}{(s+1) ! p ! l_{1}^{s+1}\left(A e^{i \Theta}\right) l_{2}^{p}\left(A e^{i \Theta}\right)}+\right. \\
+\frac{a_{2}(p+1) l_{2}\left(A e^{i \Theta}\right)\left\|F^{(s, p+1)}\left(A e^{i \Theta}\right)\right\|}{s !(p+1) ! l_{1}^{s}\left(A e^{i \Theta}\right) l_{2}^{p+1}\left(A e^{i \Theta}\right)}+ \\
\left.+\frac{\left\|F^{(s, p)}\left(A e^{i \Theta}\right)\right\|}{s ! p ! l_{1}^{s}\left(A e^{i \Theta}\right) l_{2}^{p}\left(A e^{i \Theta}\right)}\left(\frac{s\left(-u_{1}^{\prime}(t)\right)^{+}}{l_{1}\left(A e^{i \Theta}\right)}+\frac{p\left(-u_{2}^{\prime}(t)\right)^{+}}{l_{2}\left(A e^{i \Theta}\right)}\right)\right\} \leq \\
\leq g(t)\left(\max _{s+p \leq N}\left\{a_{1}(s+1) l_{1}\left(A e^{i \Theta}\right)+a_{2}(p+1) l_{2}\left(A e^{i \Theta}\right)\right\}+\right. \\
\left.+\max _{s+p \leq N}\left\{\frac{s\left(-u_{1}^{\prime}(t)\right)^{+}}{l_{1}\left(A e^{i \Theta}\right)}+\frac{p\left(-u_{2}^{\prime}(t)\right)^{+}}{l_{2}\left(A e^{i \Theta}\right)}\right\}\right)= \\
=g(t)(\beta(t)+\gamma(t)),
\end{gathered}
$$

with

$$
\begin{gathered}
\beta(t)=\max _{s+p \leq N}\left\{a_{1}(s+1) l_{1}\left(A e^{i \Theta}\right)+a_{2}(p+1) l_{2}\left(A e^{i \Theta}\right)\right\}, \\
\gamma(t)=\max _{s+p \leq N}\left\{\frac{s\left(-u_{1}^{\prime}(t)\right)^{+}}{l_{1}\left(A e^{i \Theta}\right)}+\frac{p\left(-u_{2}^{\prime}(t)\right)^{+}}{l_{2}\left(A e^{i \Theta}\right)}\right\} .
\end{gathered}
$$

Then, $\frac{d}{d t} \ln g(t) \leq \beta(t)+\gamma(t)$ and

$$
g(t) \leq g(0) \exp \int_{0}^{t}(\beta(\tau)+\gamma(\tau)) d \tau
$$

because $g(0) \neq 0$. But, one has $r^{*} A=R$. It follows from (3.14) and (3.12) that

$$
\begin{gathered}
\ln \max \left\{\frac{\left\|F^{(s, p)}\left(R e^{i \Theta}\right)\right\|}{s ! p ! l_{1}^{s}\left(R e^{i \Theta}\right) l_{2}^{p}\left(R e^{i \Theta}\right)}: s+p \leq N\right\} \leq \ln \max \left\{\frac{\left\|F^{(s, p)}(\mathbf{0})\right\|}{s ! p ! l_{1}^{s}(\mathbf{0}) l_{2}^{p}(\mathbf{0})}: s+p \leq N\right\}+ \\
+\int_{0}^{r^{*}} \max _{s+p \leq N}\left\{a_{1}(s+1) l_{1}\left(A \tau e^{i \Theta}\right)+a_{2}(p+1) l_{2}\left(A \tau e^{i \Theta}\right)\right\} d \tau+ \\
\quad+\int_{0}^{r^{*}} \max _{s+p \leq N}\left\{\frac{s\left(-u_{1}^{\prime}(\tau)\right)^{+}}{l_{1}\left(A \tau e^{i \Theta}\right)}+\frac{p\left(-u_{2}^{\prime}(\tau)\right)^{+}}{l_{2}\left(A \tau e^{i \Theta}\right)}\right\} d \tau .
\end{gathered}
$$

Inequality (3.14) is true.

Proposition 3.1. Let $\mathbf{L}\left(R e^{i \Theta}\right)$ be a positive continuously differentiable function in each variable $r_{k}, k \in$ $\{1,2\},|R|<1, \Theta \in[0,2 \pi]^{2}$. If the function $\mathbf{L}$ obeys inequality (2.1) and an analytic vector-function $F: \mathbb{B}^{2} \rightarrow \mathbb{C}^{2}$ has bounded $\mathbf{L}$-index $N=N\left(F, \mathbf{L}, \mathbb{B}^{2}\right)$ in joint variables and there exists $C>0$ such that the function $\mathbf{L}$ satisfies the inequality

$$
\sup _{|R|<1} \max _{t \in\left[0, r_{*}\right]} \max _{\Theta \in[0,2 \pi]^{2}} \max _{1 \leq j \leq 2} \frac{\left(-\left(u_{j}(t, R, \Theta)\right)_{t}^{\prime}\right)^{+}}{\frac{r_{j}}{r^{*}} l_{j}^{2}\left(\frac{t}{r^{*}} R e^{i \Theta}\right)} \leq C
$$


and

$$
\varlimsup_{|R| \rightarrow 1-0} \frac{\ln \max \left\{\|F(z, w)\|:(z, w) \in \mathbb{T}^{2}(\mathbf{0}, R)\right\}}{\max _{\Theta \in[0,2 \pi]^{2}} \int_{0}^{1}\left\langle R, \mathbf{L}\left(\tau, R e^{i \Theta}\right)\right\rangle d \tau} \leq(C+1) N+1 .
$$

Proof. If the function $\mathbf{L}$ satisfies inequality (2.1), then

$$
\max _{\Theta \in[0,2 \pi]^{2}} \int_{0}^{1}\left\langle R, \mathbf{L}\left(\tau R e^{i \Theta}\right)\right\rangle d \tau \longrightarrow+\infty, \text { as }|R| \longrightarrow 1-0 .
$$

We put $\widetilde{\beta}(t)=\sum_{j=1}^{2} a_{j} l_{j}\left(A t e^{i \Theta}\right)$. If in addition (3.15) holds, then for some $s^{*}, p^{*}, s^{*}+p^{*} \leq N$ and $\widetilde{s}, \widetilde{p}, \widetilde{s}+\widetilde{p} \leq N$,

$$
\begin{gathered}
\frac{\gamma(t)}{\widetilde{\beta}(t)}=\frac{\frac{s^{*}\left(-u_{1}^{\prime}(t)\right)^{+}}{l_{1}\left(A t e^{i \Theta}\right)}+\frac{p^{*}\left(-u_{2}^{\prime}(t)\right)^{+}}{l_{2}\left(A t e^{i \Theta}\right)}}{\sum_{j=1}^{2} a_{j} l_{j}\left(A t e^{i \Theta}\right)} \leq s^{*} \frac{\left(-u_{1}^{\prime}(t)\right)^{+}}{a_{1} l_{1}^{2}\left(A t e^{i \Theta}\right)}+p^{*} \frac{\left(-u_{2}^{\prime}(t)\right)^{+}}{a_{2} l_{2}^{2}\left(A t e^{i \Theta}\right)} \leq \\
\leq\left(s^{*}+p^{*}\right) C \leq N C
\end{gathered}
$$

and

$$
\begin{gathered}
\frac{\beta(t)}{\widetilde{\beta}(t)}=\frac{a_{1}(\widetilde{s}+1) l_{1}\left(A t e^{i \Theta}\right)+a_{2}\left(p^{*}+1\right) l_{2}\left(A t e^{i \Theta}\right)}{\sum_{j=1}^{2} a_{j} l_{j}\left(A t e^{i \Theta}\right)}=1+\frac{a_{1} \widetilde{s} l_{1}\left(A t e^{i \Theta}\right)}{a_{1} l_{1}\left(A t e^{i \Theta}\right)}+ \\
+\frac{a_{2} \widetilde{p} l_{2}\left(A t e^{i \Theta}\right)}{a_{2} l_{2}\left(A t e^{i \Theta}\right)} \leq 1+\widetilde{s}+\widetilde{p} \leq 1+N .
\end{gathered}
$$

But, $\left\|F\left(A t e^{i \Theta}\right)\right\| \leq g(t) \leq g(0) \exp \int_{0}^{t}(\beta(\tau)+\gamma(\tau)) d \tau$ and $r^{*} A=R$. Put $t=r^{*}$. In view of (3.17), we have

$$
\begin{gathered}
\ln \max \left\{\|F(z, w)\|:(z, w) \in \mathbb{T}^{2}(\mathbf{0}, R)\right\}=\ln \max _{\Theta \in[0,2 \pi]^{2}}\left\|F\left(R e^{i \Theta}\right)\right\| \leq \\
\leq \ln \max _{\Theta \in[0,2 \pi]^{2}} g\left(r^{*}\right) \leq \ln g(0)+\max _{\Theta \in[0,2 \pi]^{2}} \int_{0}^{r^{*}}(\beta(\tau)+\gamma(\tau)) d \tau \leq \\
\leq \ln g(0)+(N C+N+1) \max _{\Theta \in[0,2 \pi]^{2}} \int_{0}^{r^{*}}(\widetilde{\beta}(\tau)) d \tau= \\
=\ln g(0)+(N C+N+1) \max _{\Theta \in[0,2 \pi]^{2}} \int_{0}^{r^{*}} \sum_{j=1}^{2} a_{j} l_{j}\left(A \tau e^{i \Theta}\right) d \tau= \\
=\ln g(0)+(N C+N+1) \max _{\Theta \in[0,2 \pi]^{2}} \int_{0}^{r^{*}} \sum_{j=1}^{2} \frac{r_{j}}{r} l_{j}\left(\frac{\tau}{r^{*}} R e^{i \Theta}\right) d \tau= \\
=\ln g(0)+(N C+N+1) \max _{\Theta \in[0,2 \pi]^{2}} \int_{0}^{1} \sum_{j=1}^{2} r_{j} l_{j}\left(\tau R e^{i \Theta}\right) d \tau .
\end{gathered}
$$

Then, (3.16) is true. The Proposition 3.1 is proved.

Proposition 3.2. Let $\mathbf{L}\left(R e^{i \Theta}\right)$ be a positive continuously differentiable function in each variable $r_{k}, k \in$ $\{1,2\},|R|<1, \Theta \in[0,2 \pi]^{2}$. If the function $\mathbf{L}$ obeys inequality (2.1) and an analytic vector-function $F: \mathbb{B}^{2} \rightarrow \mathbb{C}^{2}$ has bounded $\mathbf{L}$-index $N=N(F, \mathbf{L})$ in joint variables and

$$
r^{*}\left(-\left.\left(u_{j}(t, R, \Theta)\right)_{t}^{\prime}\right|_{t=r^{*}}\right)^{+} /\left(r_{j} l_{j}^{2}\left(R e^{i \Theta}\right)\right) \longrightarrow 0
$$


for all $\Theta \in[0,2 \pi]^{2}, j \in\{1,2\}$, with $|R| \longrightarrow 1-0$, then

$$
\varlimsup_{|R| \longrightarrow 1-0} \frac{\ln \max \left\{\|F(z, w)\|:(z, w) \in \mathbb{T}^{2}(\mathbf{0}, R)\right\}}{\max _{\Theta \in[0,2 \pi]^{2}} \int_{0}^{1}\left\langle R, \mathbf{L}\left(\tau, R e^{i \Theta}\right)\right\rangle d \tau} \leq N+1 .
$$

If $\mathbf{L}(z, w)=\mathbf{L}\left(r_{1}, r_{2}\right)=\mathbf{L}(R)$, then (3.18) can be rewritten in another form.

Corollary 3.2. Let $\mathbf{L}(R)$ be a positive continuously differentiable function in each variable $r_{k}, k \in$ $\{1,2\},|R|<1$. If the function $\mathbf{L}$ obeys inequality (2.1) and an analytic vector-function $F: \mathbb{B}^{2} \rightarrow \mathbb{C}^{2}$ has bounded $L$-index $N=N(F, \mathbf{L})$ in joint variables and for each $j \in\{1,2\}$

$$
\frac{\left\langle R, \nabla l_{j}(R)\right\rangle}{r_{j} l_{j}^{2}(R)} \longrightarrow 0
$$

with $|R| \longrightarrow 1-0$, then

$$
\varlimsup_{|R| \rightarrow 1-0} \frac{\ln \max \left\{\|F(z, w)\|:(z, w) \in \mathbb{T}^{2}(\mathbf{0}, R)\right\}}{\int_{0}^{1}\langle R, \mathbf{L}(\tau R)\rangle d \tau} \leq N+1,
$$

where $\nabla l_{j}(R)=\left(\frac{\partial l_{1}(R)}{\partial r_{1}}, \frac{\partial l_{2}(R)}{\partial r_{2}}\right)$.

The main result in this section is following:

Theorem 3.4. Let $\mathbf{L}(R)=\left(l_{1}(R), l_{2}(R)\right), l_{j}(R)$ be a positive continuously differentiable non-decreasing function in each variable $r_{k}, k \in\{1,2\},|R|<1$. If the function $L$ obeys inequality (1) and an analytic vector-function $F: \mathbb{B}^{2} \rightarrow \mathbb{C}^{2}$ has bounded $L$-index $N=N(F, \mathbf{L})$ in joint variables, then

$$
\varlimsup_{|R| \longrightarrow 1-0} \frac{\ln \max \left\{\|F(z, w)\|:(z, w) \in \mathbb{T}^{2}(\mathbf{0}, R)\right\}}{\int_{0}^{1}\langle R, \mathbf{L}(\tau R)\rangle d \tau} \leq N+1 .
$$

Proof. Note that $\mathbf{L}\left(R e^{i \Theta}\right) \equiv \mathbf{L}(R)$ in this theorem. Since $l_{j}(R)$ is a positive continuously differentiable non-decreasing function and $u_{j}(t)=u_{j}(t, R)=l_{j}\left(\frac{t R}{r^{*}}\right)$, one has $\left(u_{j}(t, R)\right)_{t}^{\prime} \leq 0$. Therefore, we obtain $r^{*}\left(-\left.\left(u_{j}(t, R)\right)_{t}^{\prime}\right|_{t=r^{*}}\right)^{+} /\left(r_{j} l_{j}^{2}(R)\right)=0$. Thus, condition (3.18) is satisfied. Thus, the theorem is a direct consequence of the Proposition 3.2.

\section{REFERENCES}

[1] V.P. Baksa: Analytic vector-functions in the unit ball having bounded L-index in joint variables. Carpathian Math. Publ. 11 (2) (2019), 213-227. doi:10.15330/cmp.11.2.213-227

[2] V.P. Baksa, A.I. Bandura, O.B. Skaskiv: Analogs of Fricke's theorems for analytic vector-valued functions in the unit ball having bounded L-index in joint variables. submitted to Proceedings of IAMM of NASU.

[3] V.P. Baksa, A.I. Bandura, O.B. Skaskiv: Analogs of Hayman's theorem and of logarithmic criterion for analytic vectorvalued functions in the unit ball having bounded $\mathbf{L}$-index in joint variables. submitted to Matematica Slovaca.

[4] A. I. Bandura, O. B. Skaskiv: Analytic functions in the unit ball of bounded L-index: asymptotic and local properties. Mat. Stud. 48 (1) (2017), 37-73. doi: 10.15330/ms.48.1.37-73.

[5] A. Bandura, O. Skaskiv: Sufficient conditions of boundedness of L-index and analog of Hayman's Theorem for analytic functions in a ball. Stud. Univ. Babeş-Bolyai Math. 63 (4) (2018), 483-501. doi:10.24193/subbmath.2018.4.06.

[6] A. Bandura, O. Skaskiv: Functions analytic in the unit ball having bounded L-index in a direction. Rocky Mountain J. Math. 49 (4) (2019), 1063-1092. doi: 10.1216/RMJ-2019-49-4-1063.

[7] A. Bandura, O. Skaskiv: Asymptotic estimates of entire functions of bounded L-index in joint variables. Novi Sad J. Math. 48 (1) (2018), 103-116. doi: 10.30755/NSJOM.06997.

[8] A. Bandura, N. Petrechko, O. Skaskiv: Maximum modulus in a bidisc of analytic functions of bounded $\mathbf{L}$-index and an analogue of Hayman's theorem. Matem. Bohem. 143 (4) (2018), 339-354. doi: 10.21136/MB.2017.0110-16.

[9] A.I. Bandura, O.B. Skaskiv, V.L. Tsvigun: Some characteristic properties of analytic functions in $\mathbb{D} \times \mathbb{C}$ of bounded L-index in joint variables. Bukovyn. Mat. Zh. 6 (1-2) (2018), 21-31. doi: 10.31861/bmj2018.01.021.

[10] A.I. Bandura, N.V. Petrechko, O.B. Skaskiv: Analytic in a polydisc functions of bounded L-index in joint variables. Mat. Stud. 46 (1) (2016), 72-80. doi: 10.15330/ms.46.1.72-80.

[11] A. Bandura, O. Skaskiv: Analytic functions in the unit ball of bounded L-index in joint variables and of bounded L-index in direction: a connection between these classes. Demonstr. Math., 52 (1) (2019), 82-87. doi: 10.1515/dema-2019-0008. 
[12] A. Bandura, O. Skaskiv: Boundedness of the L-index in a direction of entire solutions of second order partial differential equation. Acta Comment. Univ. Tartu. Math., 22 (2) (2018), 223-234. doi: 10.12697/ ACUTM.2018.22.18.

[13] A.I. Bandura, O.B. Skaskiv: Partial logarithmic derivatives and distribution of zeros of analytic functions in the unit ball of bounded L-index in joint variables. J. Math. Sci. 239 (1) (2019), 17-29. doi: 10.1007/s10958-019-04284-z.

[14] A.I. Bandura, O.B. Skaskiv: Exhaustion by balls and entire functions of bounded L-index in joint variables. Ufa Math. J. 11 (1) (2019), 100-113. doi: 10.13108/2019-11-1-100.

[15] A. Bandura, O. Skaskiv: Analog of Hayman's Theorem and its Application to Some System of Linear Partial Differential Equations. J. Math. Phys., Anal., Geom. 15 (2) (2019), 170-191. doi: 10.15407/mag15.02.170.

[16] M.T. Bordulyak: On the growth of entire solutions of linear differential equations. Mat. Stud. 13 (2) (2000), $219-223$.

[17] M.T. Bordulyak, M.M. Sheremeta: Boundedness of l-index of analytic curves. Mat. Stud. 36 (2) (2011), $152-161$.

[18] L.F. Heath: Vector-valued entire functions of bounded index satisfying a differential equation. Journal of Research of NBS 83B (1) (1978), 75-79.

[19] F. Nuray, R.F. Patterson: Vector-valued bivariate entire functions of bounded index satisfying a system of differential equations. Mat. Stud. 49 (1) (2018), 67-74. doi: 10.15330/ms.49.1.67-74.

[20] R. Roy, S.M. Shah: Growth properties of vector entire functions satisfying differential equations. Indian J. Math. 28 (1) (1986), 25-35.

[21] R. Roy, S.M. Shah: Vector-valued entire functions satisfying a differential equation. J. Math. Anal. Appl. 116 (2) (1986), 349-362.

[22] M.N. Sheremeta, A.D. Kuzyk: Logarithmic derivative and zeros of an entire function of bounded l-index. Sib. Math. J. 33 (2) (1992), 304-312. doi:10.1007/BF00971102.

[23] M. Sheremeta: Boundedness of $l-M$-index of analytic curves. Visnyk Lviv Un-ty. Ser. Mech.-Math. Iss. 75 (2011), 226-231.

IVAN FRANKO NATIONAL UNIVERSITY OF LVIV

DEPARTMENT OF MECHANICS AND MATHEMATICS

1 UNIVERSYTETSKA STREET, 79000, LVIV, UKRAINE

Email address: vitalinabaksa@gmail.com

IVANO-FRANKIVSK NATIONAL TECHNICAL UNIVERSITY OF OIL AND GAS

DEPARTMENT OF ADVANCED MATHEMATICS

15 KARPATSKA STREET, 76019, IVANO-FRANKIVSK, UKRAINE

ORCID: 0000-0003-0598-2237

Email address: andriykopanytsia@gmail.com

IVAN FRANKO NATIONAL UNIVERSITY OF LVIV

DEPARTMENT OF MECHANICS AND MATHEMATICS

1 UNIVERSYTETSKA STREET, 79000, LVIV, UKRAINE

ORCID: 0000-0001-5217-8394

Email address: olskask@gmail.com 